九州大学学術情報リポジトリ

Kyushu University Institutional Repository

\title{
Simulation of Biochemical System with Computer Graphics
}

Koga, Daizo

Laboratory of Biochemistry, Faculty of Agriculture, Kyushu University

Aso, Yoichi

Laboratory of Biochemistry, Faculty of Agriculture, Kyushu University

Hayashi, Katsuya

Laboratory of Sericultural Chemistry, Faculty of Agriculture, Kyushu University

https://doi.org/10.5109/22866

出版情報：九州大学大学院農学研究院紀要. 19 (1)，pp. 33-47，1974-10. Kyushu University バージョン：

権利関係： 


\title{
Simulation of Biochemical System with Computer Graphics
}

\author{
Daizo Koga, Yoichi Aso and Katsuya Hayashi \\ Laboratory of Biochemistry and Laboratory of Sericultural \\ Chemistry, Faculty of Agriculture, \\ Kyushu University, Fukuoka \\ (Received July 24, 1974)
}

\begin{abstract}
The reaction and regulation mechanisms of biochemical reaction system in vivo have been recognized to be very complicated and most of these are remained unsolved. For the elucidation of reaction system in vivo numerous computation works would be required. In this connection, the simulation with computer graphics on the activation of pepsinogen and the lysozyme-inhibitor interaction as model reaction systems were attempted in expectation of the saving of turn-around time in digital computation. The results clearly show that this method is the most useful one for the analysis of biochemical system with respect to the speed-up of the computation work.
\end{abstract}

\section{INTORODUCTION}

The reaction mechanism, catalytic mechanism of enzymatic reaction and the mode of the regulation on each step reaction involved in a biochemical chain system such as glycolysis in living organisms have progressively been elucidated in consequence of an advance in biochemistry. The metabolic pathways of countless substances in vivo have been clarified up to date. It is obvious, however, that the regulation mechanism of a chain reaction system in vivo, by which homeostasis or rectified changes in the entire reaction system is attainable, was not demonstrated in quantitative sense, although the feedback (either negative or positive) control has been recognized to be the most fundamental principle in the regulation of biological system.

Recently, it has been gradually conceived that the quantitative and dynamic behavior or the regulation mechanisms of a biochemical system in vivo may not be predicted from the data observed in vivo on each isolated and single enzymatic reaction, because (i) the biochemical chain reaction system in vivo includes many interchain or intrachain connections (such as feedback system) for establishing the required unique regulation which are not observable on the isolated reaction system and (ii) consequently, the biochemical system exhibits essentially nonlinear behaviors such as time-retardation, oscillation or threshold phenomenon, which make the quantitative analysis of biochemical system exceedingly difficult.

On the analysis of complicated biochemical system, therefore, the intentional selection of a certain reaction scheme including the regulation system such as feedback system may be avoided, but all the possible schemes should be taken into the considerations and analyzed to find the real reaction pathway. The 
analysis of biochemical system based upon an assumed model scheme may be consisted of several successive steps ; (i) formulation of the rate equation from the reaction scheme with regulation system in form of simultaneous differential equations, (ii) estimation of the reaction parameters from the experimental data by data-fitting method consisted of optimization technique with nonlinear programming, (iii) simulation of dynamic behavior, especially the stability of the regulation system, with respect to the initial conditions and environmental conditions (milieu parameters) of the reaction and (iv) finally, design for further experiments on the subjected biochemical system must be made and the experiments will be carried out under the design to obtain more information for revising the model scheme.

The procedures mentioned above should be adopted for the all possible schemes. This means that the computational works focused to the simulation may take an enormous time (CPU and turn-around times). Thus, the saving of the time will be the most principal problem for the analysis of regulation mechanism of biochemical system.

Analog computer exerts its effectiveness for the simulation of relatively simple biochemical reaction system, because of easiness of patching of differential equation and of simplicity of selection of values of parameters involved in the rate equations. In many cases, biochemical system exhibits the nonlinearity and its behavior may be represented by simultaneous nonlinear differential equations. The nonlinear systems can easily be analyzed by analog computer utilizing multipliers and function generators. Furthermore, in either simulation or data-fitting method, parameters in the differential equation can simply be set at any desired value with a manual operation of the coefficient potentiometers. The computed results can directly be read on a cathode ray tube or recorded by an XY-plotter.

For complicated biochemical system, many integrators and potentiometers are required. The manual operation of the potentiometers becomes bothersome for such the cases and the limited number of equipped integrator restricts the adoptability of the analog computer for the computation of a large system containing many parameters in its rate equation. The other serious problems are arisen from a great difference between the values of parameters involved in the same rate equation. The difference of order of $10^{5}$ to $10^{8}$ in the rate constants (parameters) may generally appear in a biochemical system. Potentiometer setting at extremely small value may cause a great increase in the $\mathrm{S} / \mathrm{N}$ ratio, lowering the precision of the computed analog value.

With digital computer, a differential equation can usually be solved by numerical integration using subroutine program of Runge-Kutta-Gill or Hamming method. When the pitch of the time axis is sufficiently reduced corresponding to the value of parameters in the differential equation on the numerical integration, the desired precision may be held during the computation, even if the equation contains parameters with a great difference in their values. The reducing of pitch in the time axis, however, incidentally increases the CPU time. Furthermore, in the simulation on a number of scheme of biochemical system, turn-around time is expected to be critical regarding to the efficiency 
of the computation, since usually the changing of parameters along an adequate course may be mostly decided by experience of investigator himself with referring to the printed output.

If there is a computation system that permits the rapid turn-round time, an intervention of human judgement or experience, and an effective man/machine communication during the data processing by the machine, the simulation of biochemical system could be performed much effectively and rapidly. Computer graphics seems to be the most convenient method, while a few application programs satisfying the above requirements are available. The computer graphics provides the direct representation of computed results on a cathode ray tube, $\mathrm{man} / \mathrm{machine}$ communication, computation with the interruption and so on, resulting in the saving of CPU time and the rapid turn-around time.

The present report deals with case-study of computer graphics on the simulation of activation of pepsinogen and the lysozyme-inhibitor interaction.

\section{ARRANGEMENT OF EXPERIMENT AND SIMULATION}

The tactics adopted for research on the mechanism of activation of pepsinogen, as an example of biochemical system, is shown in Diagram 1. First of all, the time courses of the activation of pepsinogen near $\mathrm{pH} 2.5$ were experimentally followed by the appearance of the peptic activity or by spectrophotometric technique which detects the conformational changes of pepsinogen or pepsin molecules accompanying the activation (Step 1). With referring to the characteristic of the time-course such as clear lag-time and self-multiplicative

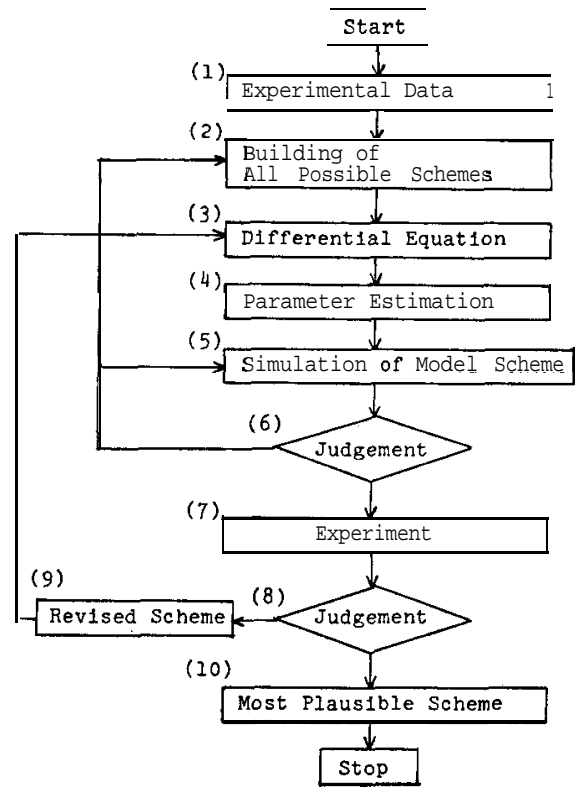

Diagram 1. Research tactics. 
pattern, all possible schemes which are capable of explaining quantitatively the characteristic were built (Step 2). For each scheme, the differential equations were formulated at Step 3 and the values of parameters involved in the rate equation were estimated by digital computer using optimization technique with nonlinear programming (Step 4). The detailed procedures included in Steps 1 to 4 and some results were reported in the previous paper (Hayashi et al., 1973). The simulation of a selected one from all possible activation schemes with the initial conditions, values of parameters and $\mathrm{pH}$ values of the activation medium have been performed efficiently with computer graphics (Step 5). Then, the adoptability of model scheme was judged at Step 6 . When the selected scheme could not explain the experimental characteristic, the other one was selected and simulated. When the selected scheme was counted to be one of the possible and plausible schemes by judgement, the predicted behaviors of the reaction scheme by the simulation technique should be supported by experimentally obtained evidences (Step 7). If the predicted behaviors can experimentally be reproduced, the subjected scheme will be registered as the most plausible scheme (Step 10). When this was unsuccessful, the some parts of the scheme should be revised (Step 9) and returned to Step 3 to repeat the successive steps.

\section{SIMULATION WITH COMPUTER GRAPHICS}

The flow chart of the simulation of the reaction scheme by computer graphics is shown in Diagram 2. For computer graphics, FACOM 230-60 and a graphic display unit GDP F6233A with a cathode ray tube (CRT) installed in Computer Center, Kyushu University were used. The principal feature of the flow chart is to include the interruption and input by a console typewriter, permitting the efficient man/machine communication or human judgement with referring to the displayed patterns during the execution or data processing. The term interruption means an instruction to a computer to suspend the execution of a program and to select the statement to be executed next within the program. The external signal for giving the instruction is generated by handling of a light-pen, function keys or auxiliary switch (see Diagram 3). The control of the execution of a program under human recognition and judgement can be achieved by this function.

First, the title or the subject of the simulation is indicated on CRT (Diagram 2, Step 1). Then, the specification whether the input of experimental data is necessary or not, is given at Step 2. The selection of a certain scheme from a set of possible reaction schemes is done at Step 3 and the selected scheme is displayed on CRT (Step 4). The initial conditions and parameters of differential equations derived from the selected scheme are fed (Step 5) and the differential equations are solved by numerical integration using Runge-Kutta-Gill method (Step 6). Calculated time-courses of the reaction are displayed on CRT and, if it is necessary, the experimental data are also indicated on CRT at the same time.

When several curves are simultaneously displayed on CRT, a symbol with number is put to each curve to distinguish the reaction species and the specific 


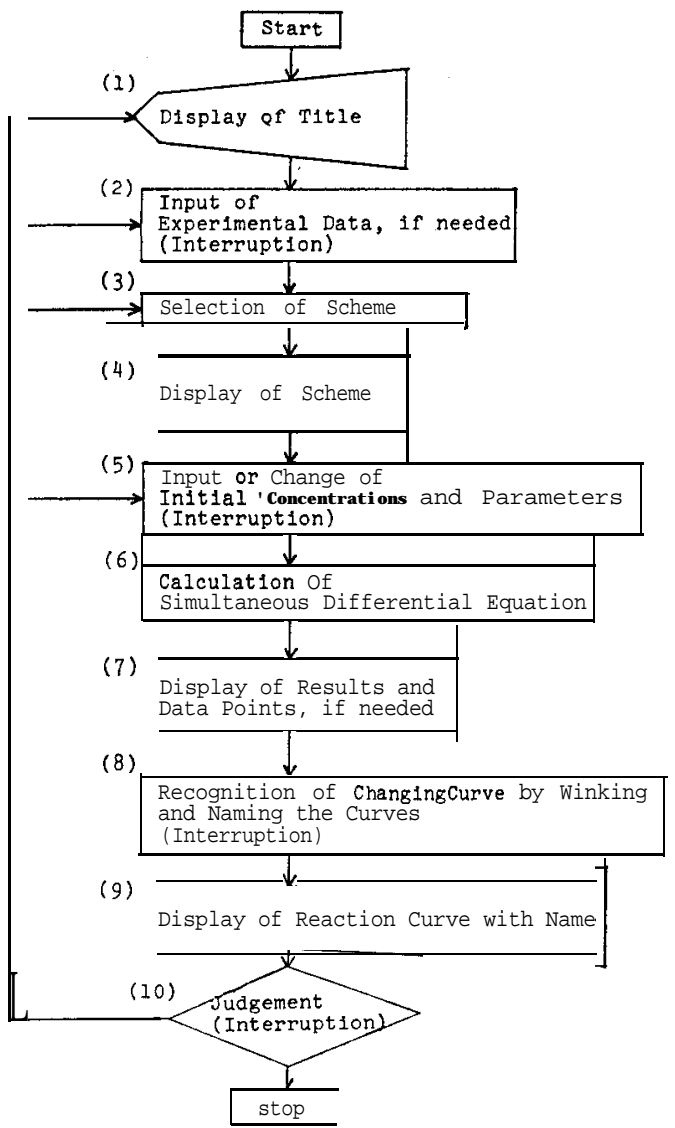

Diagram 2 . Flow chart of simulation with computer graphics.

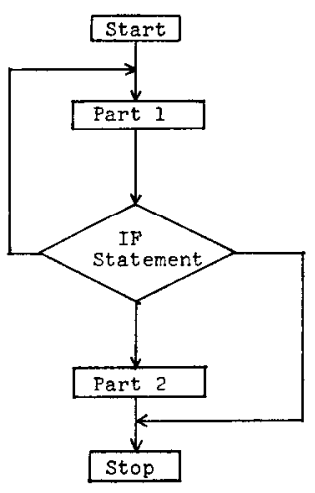

(I)

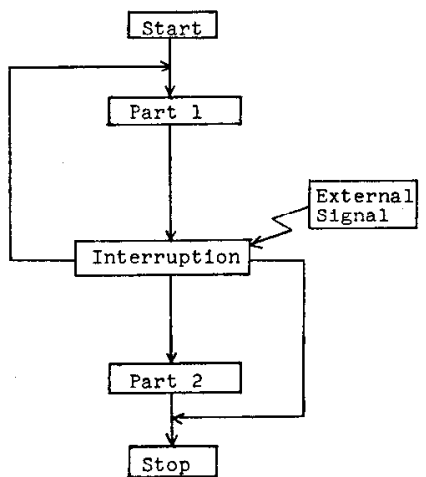

(II)

Diagram 3. Illustrated comparison between usual program (I) and program including the interruption (II). 
curve to be examined in detail is winked (Steps 8 and 9). The judgement on continuation or stop of the simulation is done at Step 10. For the case of continuation, it is necessary to indicate the junction and the destination of do-loop under the human judgement. The return to Steps 1 to 5 can be directed by means of light-pen on CRT.

\section{RESULTS}

\section{Activation of pepsinogen}

\section{Model schemes of activation}

It was reported in the previous paper (Hayashi et al., 1973) that about 25 schemes were subjected to the simulation by analog computer to examine their adoptabilities for the analysis of experimental data. Consequently, several schemes were found to be possible ones, explaining the experimentally obtained characteristic on the activation of pepsinogen. In the subsequent experiment, the activation process of pepsinogen was followed by gelfiltration and it was found that there were only three molecular species with respect to molecular weight, i. e., pepsinogen species, pepsin species and dimeric form of pepsinogen and pepsin (Koga, unpublished data). At the present time, seven schemes are postulated according to the results of analog computer simulation and newly obtained experimental findings (Table 1). Schemes 1 to 3 are formulated with the intention to analyzing the change in the reaction species with regard to molecular weight during the activation. Schemes 4 and 5 are selected as the most plausible ones from the analog computer simulation and had been denoted

Table 1. Postulated schemes for activation of pepsinogen.

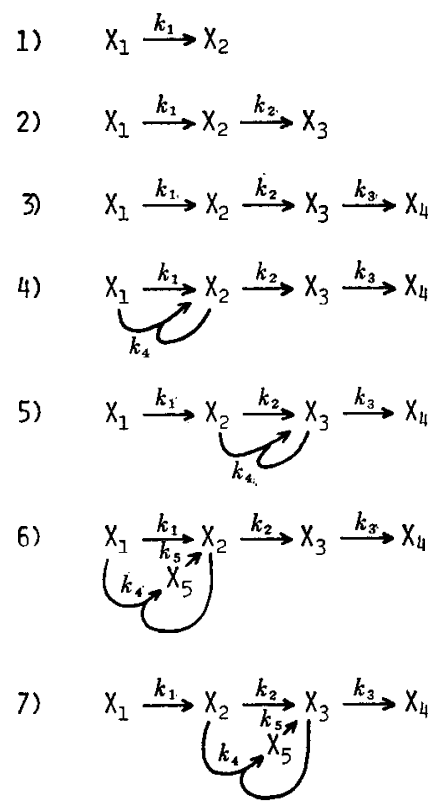


by Schemes 2-2 and 3-2 respectively, in the previous paper. Schemes 6 and 7 are derived from Schemes 4 and 5 respectively, by inserting the dimeric reaction species in the looped step.

Rate equation

The simulation of Scheme 7 will be described here as an example, since this scheme seems to be most reliable one. The scheme displayed on CRT is shown in Fig. 1. The lines between reaction species were given in the input as specified vectors. The $\mathrm{X}_{1}$ and $\mathrm{X}_{4}$ represent the pepsinogen and pepsin molecules respectively. The intermediate $X_{2}$ is assumed to have the same molecular weight as pepsinogen and inactive, $X_{3}$ has the same size as pepsin and active, $X_{5}$ is the dimeric intermediate. The $X_{6}$ indicates the sum of $X_{1}$ and $X_{2}$ that have same size and $X_{7}$ the sum of $X_{3}$ and $X$,. The peptic activity is represented conveniently by the amount of $X_{7}$ with assuming that $X_{3}$ and $X_{4}$ have the same specific activity. The $k_{1}$ indicates the rate constant of $\mathrm{i}$-th step. Except $k_{4}$ $\left(\mathrm{M}^{-1} \sec ^{-1}\right)$, others are first-order rate constant $\left(\sec ^{-1}\right)$.

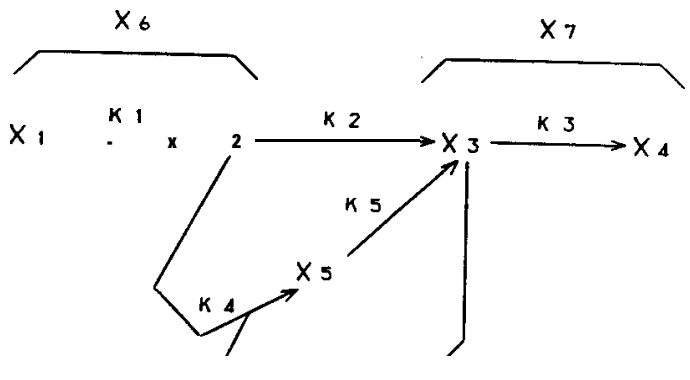

Fig. 1. Scheme 7 displayed on CRT.

The differential equations derived from Scheme 7 are :

$$
\begin{aligned}
& \frac{d X}{d t}=-k_{1} X_{1} \\
& \frac{d X_{2}}{d t}=k_{1} X_{1}-k_{2} X_{2}-k_{4} X_{2} X_{3} \\
& \frac{d X_{3}}{d t}=k_{2} X_{2}+2 k_{5} X_{5}-k_{3} X_{3}-k_{4} X_{2} X_{3} \\
& \frac{d X_{4}}{d t}=k_{3} X_{3} \\
& \frac{d X_{5}}{d t}=k_{4} X_{2} X_{3}-k_{5} X_{5}
\end{aligned}
$$

where italic $X_{1} \sim X_{7}$ indicate the amount of the reaction species, The values of parameters estimated by numerical integration are $; k_{1}=2.5 \times 10^{-3} \mathrm{sec}^{-1}, k_{2}=5$ $\times 10^{-3}$ see- $, k_{3}=1 \times 10^{-3} \mathrm{sec}^{-1}, k_{4}=9.08 \times 10^{-2} \mathrm{M}^{-1} \mathrm{sec}^{-1}$ and $k_{5}=9.97 \times 10^{-2} \mathrm{sec}^{-1}$. These values were estimated from the experimental data at $\mathrm{pH} 2.85$. 


\section{Simulation with initial concentration}

The typical patterns are shown in Figs. 2, 3 and 4. All figures are the photograph of the figure displayed on CRT. Notching lines on time axis, for example, are bended, because of inadequate adjustment of instrument. Such the defects are not corrected since these do not alter the precision of the computed results. It is obvious that the rate of the activation depends upon the initial concentration of pepsinogen. The feature of concentration dependence of the rate is shown in Fig. 5, where the reciprocal of time corresponding to the crosspoint of $X_{6}$ and $X_{7}$ is assumed to be proportional to the rate of the activation. The initial concentration below $0.4 \%$ of pepsinogen leads a remarkable decrease in the activation rate. This may be caused from the nonlinearity of the activation system. In this simulation, the values of the initial concentration of pepsinogen were displayed on CRT and the selection of the value for computa-

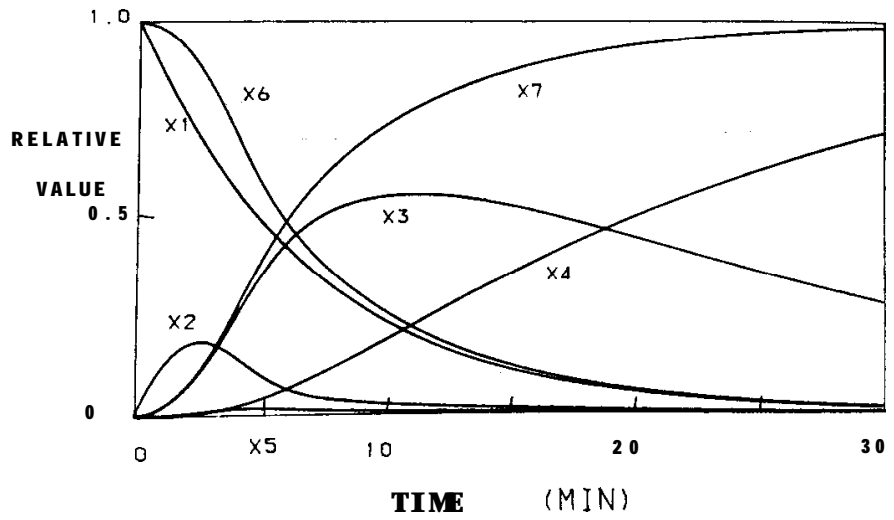

Fig. 2. Time-course of activation of pepsinogen with Scheme 7. The curves were obtained by data-fitting to experimental results at initial concentration of pepsinogen of $0.2 \%, \mathrm{pH} 2.85$ and $0^{\circ} \mathrm{C} . k_{1}=2.5 \times 10^{-3} \mathrm{sec}^{-1}, k_{2}=5 \times 10^{-3} \mathrm{sec}^{-1}, k_{3}=1 \times 10^{-3} \mathrm{sec}^{-1}, k_{4}=$ $9.08 \times 10^{-2} M^{-1} \mathrm{sec}^{-1}, k_{5}=9.97 \times 10^{-2} \mathrm{sec}^{-1}$.

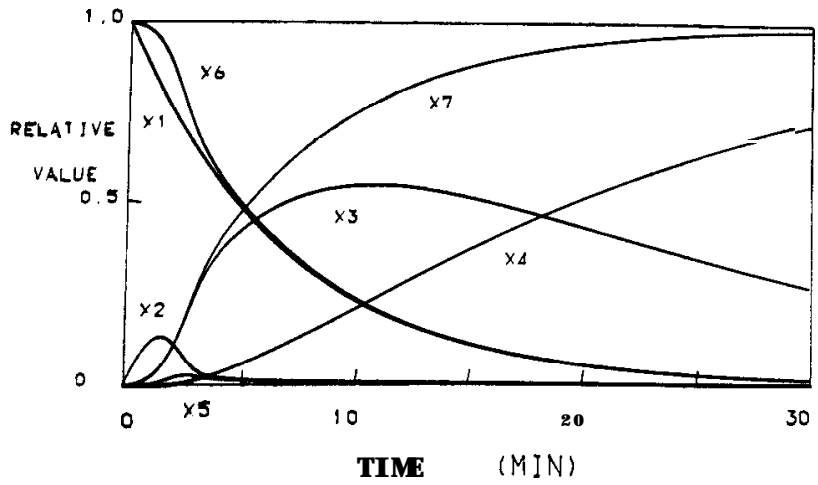

Fig. 3. Simulated time-course with Scheme 7. Initial concentration of pepsinogen was $1 \%$ (five-time higher than that in Fig. 2). Other parameters were the same. 


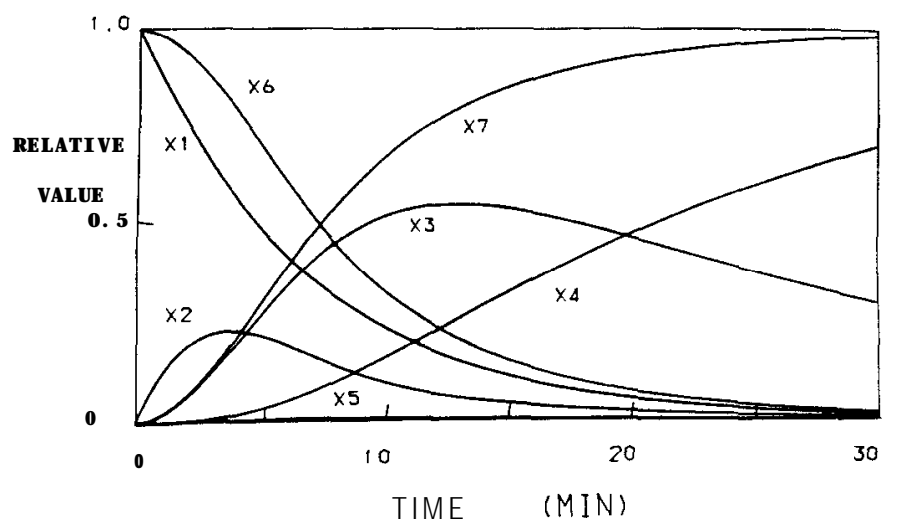

Fig. 4. Simulated time-course with Scheme 7. Initial concentration of pepsinogen was $0.04 \%$ (one-fifth of that in Fig. 2). Other parameters were the same as in Fig. 2.

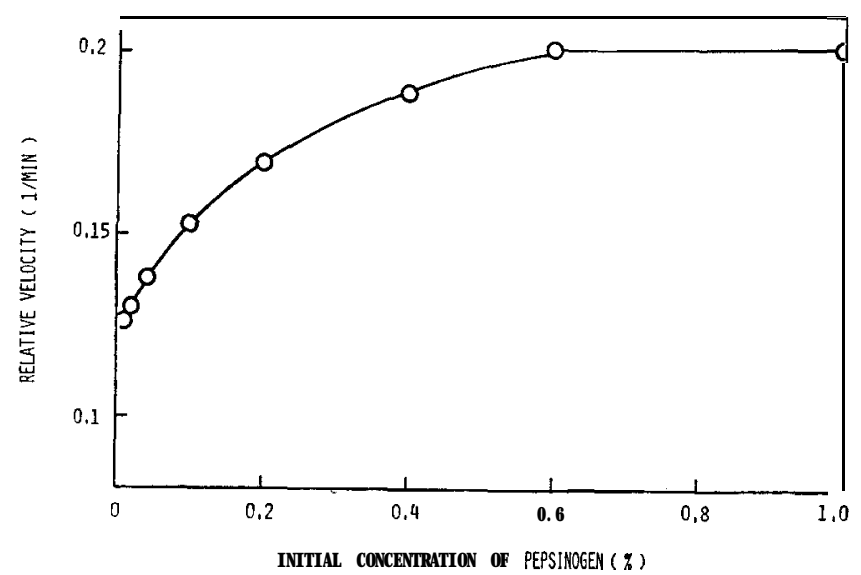

Fig. 5. Relation between initial concentration of pepsinogen and the reciprocal time (relative activation time) simulated with Scheme 7.

tion was pointed out by a light-pen. Otherwise, the initial concentrations were given by input through a console typewriter.

\section{Simulation with parameter}

The changes in the reaction time-course with alteration of values of parameters are shown in Figs. 6 and 7 as expamles. Doubling or halving of parameters, $k_{2} \sim k_{4}$, scarcely affects the time-course of the activation. However, only the rate constant of first step, $\mathbf{k}_{\boldsymbol{\prime}}$, has a sharp effect on the time-courses. The input of various values of parameters were made in the same way as before.

\section{pH-Dependence of activation process}

It has been well known that the rate of the activation of pepsinogen depends strongly upon the pH value of the medium (Bohak, 1973 ; Al-Janabi et al., 1972). 


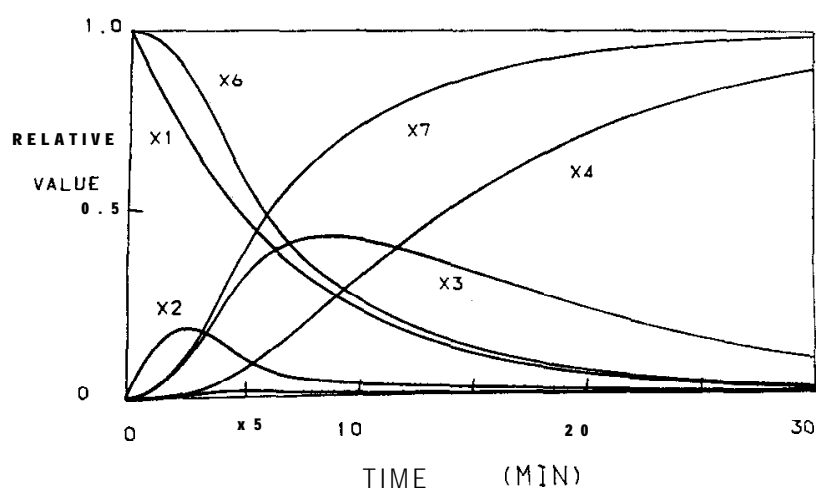

Fig. 6. Simulated time-course with Scheme 7 to examine sensitivity of rate of activation on $k_{3}$. Value of $k_{3}$ was twice higher than that in Fig. 2.

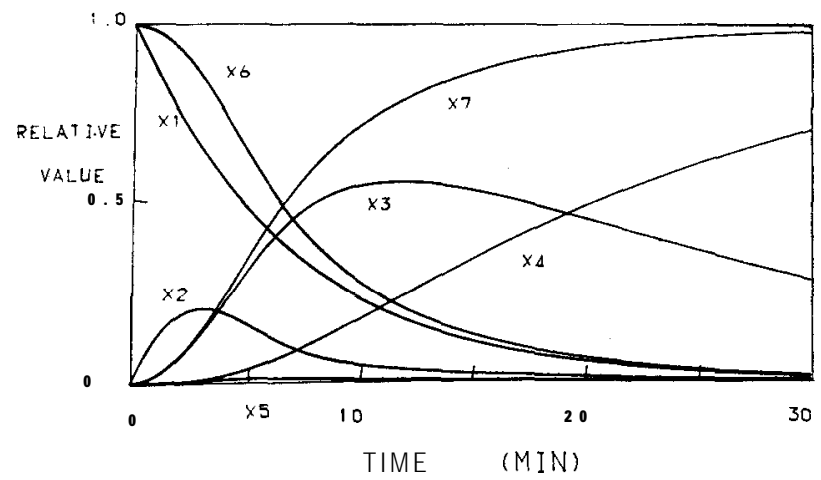

Fig. 7. Simulated time-course with Scheme 7. Value of $k_{4}$ was half of that in Fig. 2.

Consequently, the introduction of the hydrogen ion concenration into the rate equation was attempted. In this simulation, it is assumed that the rate constant of the first step is the simple function of hydrogen ion concentration $(\mathrm{H})$, i. e., $k_{1}=k^{\prime}(\mathrm{H})$. The results are shown in Figs. 8-10. Below $\mathrm{pH}$, the change in the rate was considerably small as shown in the figures. At $\mathrm{pH} 4$, the rate becomes extremely low and only about $25 \%$ of pepsin formed within 30 min incubation.

Early experimental results indicated that pepsin molecule showed multi-states with respect to its activity (Northrop, 1930), especially near neutral region. This findings suggest that the final step of the activation, $X_{3} \rightarrow X_{4}$, would have equilibrium nature and would be represented by $X_{3} \rightarrow X_{4}$. It is reasonable, further, to assume that this equilibrium step is $\mathrm{pH}$-dependent and the equilibrium constant is a function of $\mathrm{pH}$ value, $\mathrm{k}=k_{3} / k_{-3}=f(\mathrm{pH})$. Thus, Scheme 8 is postulated. The results of the simulation of Scheme 8 with changing the $\mathrm{pH}$ value will be reported in a separate paper. 


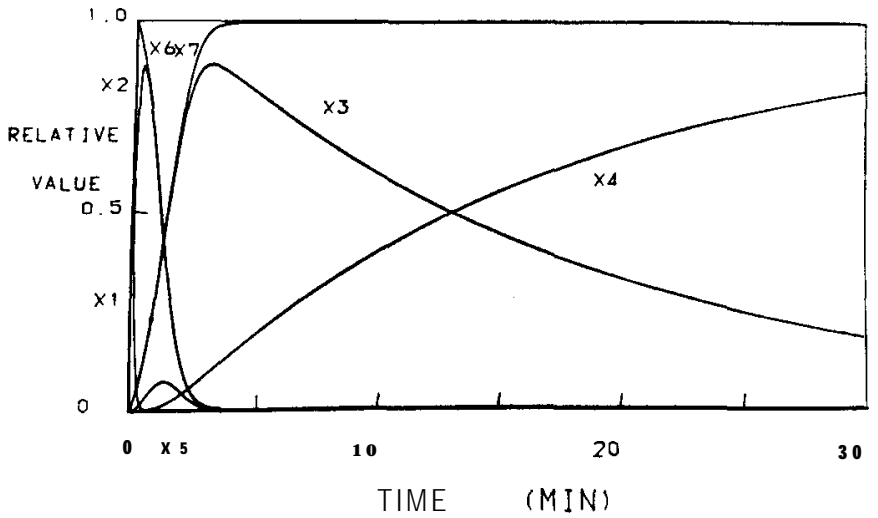

Fig. 8. Simulated time-course with Schem 7. Value of $k_{1}$ was $1.77 \times 10^{-1}$ corresponding to activation at $\mathrm{pH} 1$.

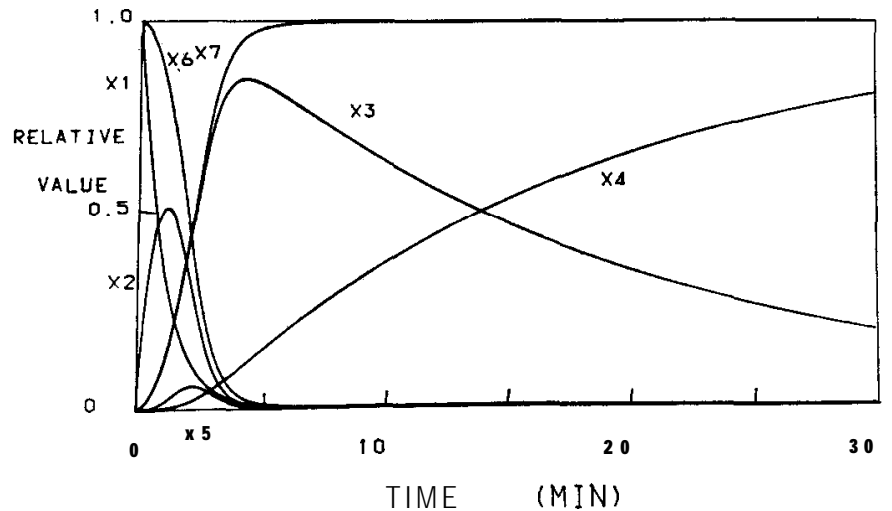

Fig. 9. Simulated time-course with Scheme 7. Value of $k_{1}$ was $1.77 \times 10^{-2}$ corresponding to $\mathrm{pH} 2$.

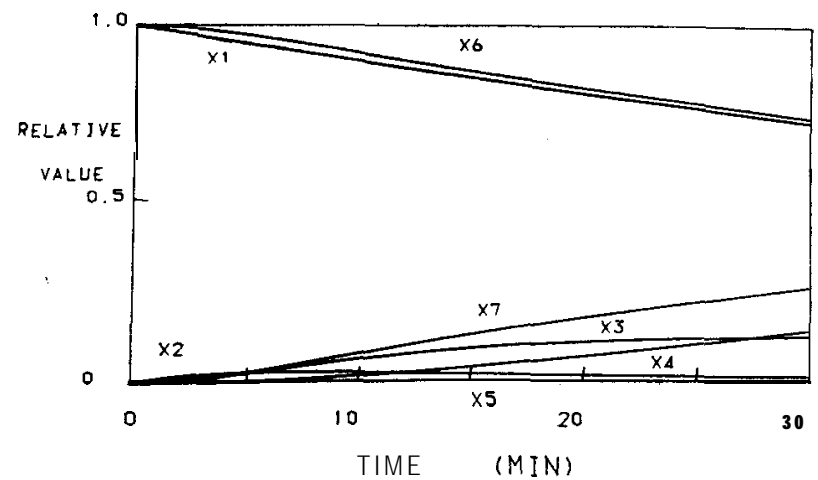

Fig. 10. Simulated tim-course with Scheme 7. Value of $k_{1}$ was $1.77 \times 10^{-4}$ corresponding to $\mathrm{pH} 4$. 


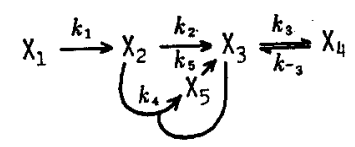

Scheme 8

\section{Comparison with experimental data}

The predicted behaviors of Scheme 7 by this simulation have been evidenced by experiments on the effect of initial concentration of pepsinogen and the $\mathrm{pH}$ value of the medium. The experimental results will also be reported on a succeeding paper.

\section{Lysozyme-inhibitor interaction}

\section{Rate equation}

It is the characteristic feature obtained experimentally on the lysozymeinhibitor (dimethylbenzylmyristylammonium chloride) interaction that the rate of the formation of lysozyme-inhibitor complex was reciprocally proportional to the initial concentration of the inhibitor (Hayashi et al., 1968a, b). Many interaction schemes were surveyed to find a possible scheme that can explain quantitatively the experimental findings. However, most of them should be rejected because of failure in fitting to the experimental data (Aso et al., 1974).

Only selected scheme at the present time that may have basic capability to explain the abnormal behavior of the lysozyme-inhibitor interacton with regard to the relation between the rate and the initial concentration of the inhibitor, may be represented by :

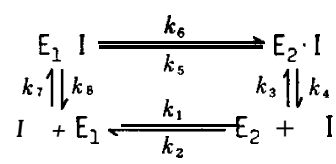

Lysozyme-inhibitor interaction

where $I$ represents the inhibitor, $E_{1}$ and $E_{2}$ the enzymes at different states, $E_{1} I$ and $E_{2} I$ the enzyme-inhibitor complexes and $k,(i=1,2, \ldots, 8)$ are the rate constants. The differential equation for this scheme may be written as :

$$
\begin{aligned}
& \frac{d X_{1}}{\mathrm{dt}}=-k_{1} X_{1}+k_{2} X_{2}-k_{7} X_{1} X_{3}+k_{8} X_{4} \\
& \frac{d X_{2}}{\mathrm{dt}}=k_{1} X_{1}-k_{2} X_{2}-k_{3} X_{2} X_{3}+k_{4} X_{5} \\
& \frac{d X_{3}}{\mathrm{dt}}=-\left(k_{7} X_{1}+k_{3} X_{2}\right) X_{3}+k_{8} X_{4}+k_{4} X_{5} \\
& \frac{d X_{4}}{d t}=-\left(k_{6}+k_{8}\right) X_{4}+k_{5} X_{5}+k_{7} X_{1} X_{3} \\
& \frac{d X_{5}}{\mathrm{dt}}=-\left(k_{4}+k_{5}\right) X_{5}+k_{6} X_{4}+k_{3} X_{2} X_{3}
\end{aligned}
$$


where $X_{1}(\mathrm{i}=1,2, \ldots, 5)$ are the concentrations at time $t$ of $\mathrm{E}, \mathrm{E}_{2}, \mathrm{I}, \mathrm{E}_{1} \mathrm{I}$ and $\mathrm{E}_{2} \mathrm{I}$.

\section{Simulation}

There may be two cases according to the selection of rate constants, since the scheme has a cycle pathway. One is a case where an equilibrium state can be attained, and the other shows steady-state but has not the solution of the differential equation with regard to the equilibrium concentrations. In the former case, the relation, $\left(k_{1} \cdot k_{3}\right) /\left(k_{2} \cdot k_{1}\right)=\left(k_{1} \cdot k_{7}\right) /\left(k_{5} \cdot k_{1}\right)$, is realized, and in the latter case, this is not seen and the equilibrium concentrations of reaction species could not be calculated. On the simulation, only the former case was analyzed. The interaction system which can attain the equilibrium state may be divided into two classes. One is a system where the inhibitor is added to the equilibrium state of $\mathrm{E}_{1} \rightleftharpoons \mathrm{E}_{2}$. This system is tentatively called "pre-equilibrium system." The other is a system where the inhibitor is added to $E_{1}$ (therefore, $\mathrm{E}_{2}$ does not exist at the initial state) and called "non pre-equilibrium system."

The simulation was performed by changing the initial concentration of the inhibitor to observe the time-course of $E_{1} I$ and $E_{2} I$ complexes with the initial concentration of the enzyme at $6.8 \times 10^{-5} \mathrm{M}$ and the initial concetrations of inhibitor at $4.512-0.564 \times 10^{-3} \mathrm{M}$. The results are shown in Figs. 11 (pre-equilibrium system) and 12 (non pre-equilibrium system). The vertical axis represents the ratio of concentration of the complex at time $t$ to the largest equilibrium concentration. Both systems exhibit nearly the same pattern, showing that the rate of the interaction is reciprocally proportional to the initial concentration of the inhibitor. The non pre-equilibrium system shows an induction period that is presumably arisen from the delay-time for the formation of $\mathrm{E}$,. These results infer that the cycle scheme subjected in this simulation has a fundamental property that can result in the abnormal dependence of interaction rate to the initial concentration of the inhibitor.

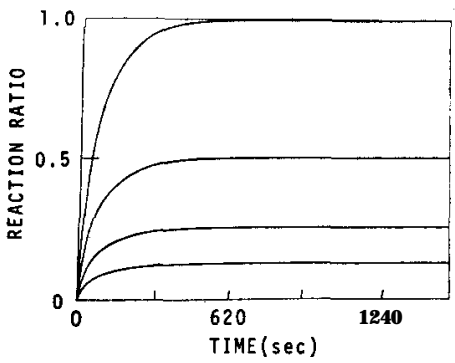

Fig. 11. Calculated time-course of $E_{1} I$ complex in pre-equilibrium system. Initial concentration of enzyme was fixed at $6.8 \times 10^{-5} \mathrm{M}$ and initial concentrations of inhibitor were from top to bottom $4.512 \times 10-3,2.256 \times 10^{-3}, 1.128 \times 10^{-3}$ and $5.64 \times 10^{-4} \mathrm{M}$. Rate constants were $1 \mathrm{X} 10^{-2} \mathrm{M}^{-1} \sec ^{-1}$ (for $k_{3}, k_{4}, k_{7}, k_{8}$ ) and $1 \mathrm{X} 10^{-2} \sec ^{-1}$ (for $k_{1}, k_{2}$. $k_{5}, k_{6}$ ). Time-course of $E_{2} I$ complex was nearly the same as that of $E_{1} I$ complex. 


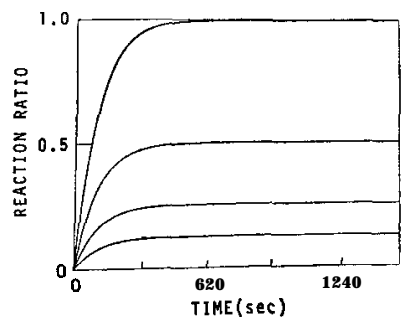

(a)

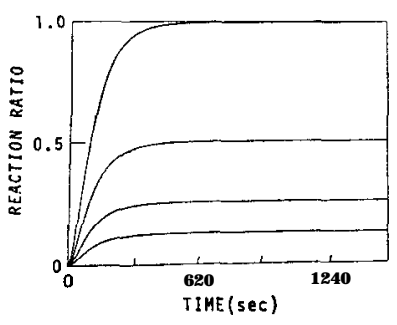

(b)

Fig. 12. Calculated time-course of $E_{1} I$ (a) and $E_{2} I$ (b) in non pre-equilibrium system. Initial concentrations and rate constants were the same as in Fig. 11.

\section{DISCUSSION}

The effective computation by digital computer may be based on the suitable man/machine communication. On such a computation, the flow of execution or data processing by the machine should be controlled by human judgement. For this purpose, it is necessary to establish such the way that (i) with the interruption, the intermediary results of the computation are presented on a display unit or as typewriting output, (ii) human judgement is made on the presented intermediary results to decide the direction of following flow of execution, (iii) based upon the judgement, new data are fed as iuput or are called from the memory and new route of the execution is dictated, (iv) the interruption is canceled and data processing is continued, (v) these steps are repeated.

Computer graphics satisfies well the above demands. Furthermore, in this technique, human judgement can be done with displayed figure in the way of pattern-recognition.

It is confirmed by the present study that for the simulation of biochemical reaction system, computer graphics is most suitable and can save the computation time to a great extent. However, a huge programming could not be avoided due to the introduction of interruption, display and dictation items. The biochemical reaction system to be analyzed in connection with the mechanism of the biochemical regulation may be consisted of a large number of elementary step, forming a network circuit. For the analysis of such complicated biochemical system, more sophisticated technique such as ADSL (FACOM application program) should be simultaneously established.

\section{REFERENCES}

Al-Janabi, J., J. A. Hartsuk and J. Tang 1972 Kinetic and Mechanism of Pepsinogen Activation. J. Biol. Chem., 247: 4628-4632

Aso, Y., D. Koga, F. Mori, K. Hayashi and M. Funatsu 1974 Kinetic Analysis of Lysozymeinhibitor Complex Formation. J.Fac. Agr., Kyushu Univ., 18: 89-106

Bohak, Z. 1973 Kinetics of Conversion of Chicken Pepsinogen to Chicken Pepsin. Eur. J. Biochem., 32 : 547-554 
Hayashi, K., M. Kugimiya, T. Imoto, M, Funatsu and C. C. Bigelow 1968a The Inhibitory Interaction of Cationic Detergents with the Active Center of Lysozyme. I. Site of Interaction. Biochemistry, 7 : 1461-1466

Hayashi, K., M. Kugimiya, T. Imoto, M. Funatsu and C. C. Bigelow 1968b The Inhibitory Interaction of Cationic Detergents with the Active Center of Lysozyme. II. The pH Dependence of the Interaction. Biochemistry, $7: 1467-1472$

Hayashi, K., D. Koga, Y. Aso, E. Hayashida, T. Kaneda, M. Funatsu and T. Matsui 1973 On the Activation of Pepsinogen. J.Fac. Agr., KyushuUniv.,18: 21-51

Northrop, J. H. 1930 Crystalline Pepsin. J. Gen. Physiol., 13: 739-766 